\title{
Identificação de Operantes Verbais Constituintes da Leitura por meio da Análise dos Movimentos dos Olhos
}

\author{
Peter Endemann ${ }^{1}$ \\ Candido Vinicius Bocaiúva Barnsley Pessôa \\ William Ferreira Perez \\ Gerson Yukio Tomanari \\ Universidade de São Paulo
}

\begin{abstract}
RESUMO - Os movimentos dos olhos produzem estímulos discriminativos para o comportamento de ler. O presente estudo teve por objetivo analisar os movimentos dos olhos de dois participantes durante a leitura de anagramas e identificar os operantes verbais envolvidos. A tarefa consistia em vocalizar uma palavra utilizando cinco letras. Na Fase 1, as letras formavam apenas uma palavra, sempre que observadas em uma mesma sequência espacial. Na Fase 2, formavam duas ou mais a partir de diferentes sequências. Na Fase 2, apesar da possibilidade de múltipla formação de palavras, as palavras vocalizadas foram aquelas formadas pela mesma sequência reforçada na fase anterior. Com base nos movimentos dos olhos, as vocalizações foram analisadas à luz dos conceitos de comportamento textual, auto-correção e intraverbal.
\end{abstract}

Palavras-chave: movimentos dos olhos; comportamento textual; comportamento intraverbal; leitura; humanos.

\section{Identifying Verbal Operants in Reading by Means of Eye Movement Analysis}

\begin{abstract}
Eye-movements produce discriminative stimuli for reading. The objective of the present study was to analyze the eye movements of two participants during anagram reading and to identify the verbal behavior involved in it. The task consisted of vocalizing a word composed of five letters. In Phase 1, the letters formed only one possible word when observed in a specific spatial sequence. In Phase 2, the letters formed two or more words via different sequences. In Phase 2, despite the fact that multiple words could be read, the vocalized ones were those formed by the same sequence reinforced in the previous phase. Based on the eye movements, vocalizations were analyzed using the concepts of textual, self-correction and intraverbal behavior.
\end{abstract}

Keywords: eye movements; textual behavior; intraverbal behavior; reading; humans.

Os movimentos dos olhos antecedem e/ou são simultâneos a uma infinidade de comportamentos (Schroeder \& Holland, 1968a, 1968b), desde comportamentos simples, como procurar ou pegar um objeto, até comportamentos refinados e específicos do ser humano, como o comportamento verbal e, mais especificamente, a leitura (Skinner, 1957).

O comportamento verbal foi descrito por Skinner (1957) como um comportamento operante (do falante) estabelecido e mantido pela mediação de outras pessoas (os ouvintes) especialmente treinadas para isso (Passos, 2007). Skinner classificou diferentes comportamentos verbais baseando-se em diferentes relações entre os estímulos antecedentes, tipos de respostas e suas consequências. Respostas verbais controladas por estímulos antecedentes verbais e por reforçadores generalizados foram classificados como comportamentos ecóicos, transcrições, ditados, textuais ou intraverbais. No que se refere aos objetivos deste trabalho, faz-se necessário especificar as características das relações que descrevem, em particular, os comportamentos textuais e intraverbais.

O comportamento textual é descrito pelas relações em que estímulos antecedentes verbais evocam respostas vocais

1 Endereço para correspondência: Av. Prof. Mello Moraes, 1721, Bloco A, Sala A-5. Cidade Universitára. São Paulo, SP. CEP. Fone (11) 81726350.E-mail peterendemann@hotmail.com que, por sua vez, são mantidas por apresentações de estímulos reforçadores generalizados. Geralmente, no comportamento textual, os estímulos antecedentes são visuais, como no caso de palavras e textos impressos, mas podem ser também táteis, como no caso do Braille. Na relação entre o estímulo antecedente e a resposta, Skinner destaca uma correspondência ponto-a-ponto (Skinner, 1957) ou, como aponta Matos (1991), uma identidade funcional. Por exemplo, diante da palavra impressa CASA, o falante vocaliza "casa". Nesse caso, existe uma correspondência entre as unidades escritas e as unidades faladas, de tal forma que cada unidade funcional do estímulo impresso controla a emissão de cada unidade da resposta de vocalização da palavra (cf. Passos, 2003). Se houver relação ponto-a-ponto entre o estímulo antecedente verbal e a resposta emitida, um membro da comunidade verbal pode apresentar um reforçador generalizado contingente à resposta. Aponta-se, entretanto, que, em se tratando de um comportamento social, provavelmente haverá intermitência nas apresentações dos reforçadores que mantêm o comportamento textual.

O comportamento intraverbal também é controlado por estímulos antecedentes verbais e por reforçamento generalizado. Porém, no caso do comportamento intraverbal, não é possível identificar uma relação de correspondência 
ponto-a-ponto entre o estímulo antecedente e a resposta. Por exemplo, diante do estímulo antecedente verbal $2+2=$, o falante emite a resposta "quatro" (Skinner, 1957).

Ao analisar a aprendizagem da leitura, Skinner (1957) aponta que unidades de controle de diferentes tamanhos podem controlar as respostas verbais no comportamento textual. De Rose (2005) destaca, ainda, a necessidade de que as unidades dispostas no texto se encadeiem em uma sequência espacial específica. No caso da língua portuguesa, os movimentos requisitados são da esquerda para a direita e de cima para baixo. Esse encadeamento se dá por meio do movimento dos olhos (no caso do Braille, por meio do movimento dos dedos).

Tarefas de leitura ocupam lugar de destaque nas pesquisas sobre rastreamento do olhar (Gompel, Fischer, Murray, \& Hill, 2007). O comportamento de participantes em tarefas de leitura tem sido vastamente estudado na literatura analítico-comportamental (por exemplo, de Rose, de Souza, \& Hanna, 1996; de Souza et al., 2009; Hübner, Gomes, \& McIvane, 2009). Kaplan e Schoenfeld (1966), por exemplo, investigaram o condicionamento operante dos movimentos dos olhos em tarefas de leitura de anagramas. No relato de sua pesquisa, esses autores citam um experimento de Rees e Israel (conforme citado por Kaplan \& Schoenfeld, 1966) realizado para estudar solução de problemas. Sem o registro dos movimentos dos olhos, Rees e Israel inicialmente ensinaram participantes a solucionar anagramas em uma sequência especifica. Posteriormente, verificaram se a seqüência que fora ensinada era utilizada também diante da apresentação de anagramas ambíguos, ou seja, que possibilitavam a formação de diferentes palavras a depender da sequência das letras. Durante o experimento, anagramas com cinco letras foram sucessivamente apresentados para os participantes. Os participantes do experimento foram divididos em um grupo experimental e um grupo controle. Para ambos os grupos, as letras estavam sempre dispostas da seguinte forma: uma no centro e as demais nos cantos do anagrama (Figura 1). Durante todo experimento, as letras dos anagramas formavam palavras sempre que ordenadas na mesma sequência de posições: partindo-se da letra do centro, em seguida a letra inferior esquerda e, daí em diante, no sentido anti-horário (sequência 3-4-5-2-1, Figura 1).

O grupo experimental passou por duas fases. A primeira fase foi composta por trinta apresentações sucessivas de distintos anagramas. A apresentação do anagrama era encerrada depois de o participante "resolvê-lo" ou decorrida a passagem de $20 \mathrm{~s}$. Os participantes do grupo controle foram submetidos somente à segunda fase.

No procedimento empregado por Rees e Israel, a cada apresentação, era requerido dos participantes apertar um botão quando encontrassem a palavra que era formada pelas cinco letras. Após apertar o botão, os participantes deveriam vocalizar a palavra-solução. Os experimentadores registraram o tempo entre a apresentação do anagrama e a resposta de apertar o botão e, também, a palavra vocalizada. Imediatamente após a exposição aos trinta anagramas, começava a segunda fase. Nesta segunda fase, dez anagramas ambíguos foram sucessivamente apresentados. Diante destes anagramas, os participantes do grupo experimental vocalizaram a palavra-solução formada pela sequência presente na primeira fase em 94,6\% das apresentações. Os participantes do grupo controle vocalizaram estas palavras apenas em 47,3\%.

Kaplan e Schoenfeld (1966) também utilizaram anagramas de cinco letras dispostas do mesmo modo que em Rees e Israel (conforme citado por Kaplan \& Schoenfeld, 1966) (Figura 1). Na Fase 1 do experimento, as letras dos anagramas formavam uma só palavra, sempre quando lidas na mesma sequência. Na Fase 2, as letras dos anagramas formavam também uma só palavra-solução, só que em uma sequência diferente. Por fim, na Fase 3, a sequência da única solução foi novamente modificada. Os anagramas foram apresentados por meio de um projetor e os experimentadores registraram os movimentos dos olhos dos participantes por meio de um equipamento fotográfico cuja velocidade era de 13,6 quadros por segundo.
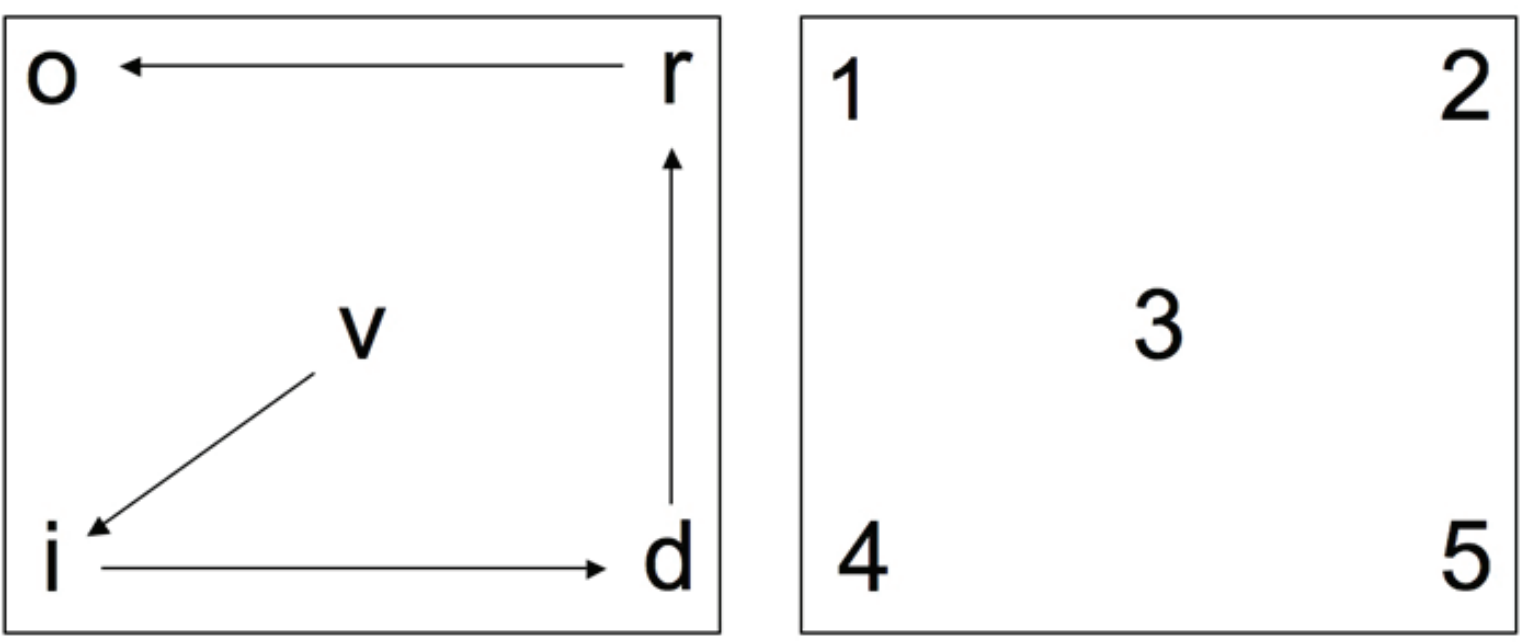

Figura 1. Representação esquemática da disposição em xis de letras que formavam os anagramas do experimento de Rees e Israel (conforme citado por Kaplan \& Schoenfeld, 1966). A figura da esquerda mostra um anagrama com letras e a figura da direita mostra um índice numérico para cada posição de letra. Na figura, a palavra VIDRO é formada na sequência 3-4-5-2-1. No experimento original, outras letras compunham os anagramas e formavam palavras em inglês. 
Kaplan e Schoenfeld (1966) verificaram que, após algumas apresentações, os movimentos dos olhos dos participantes que resolviam a tarefa mais rapidamente (respostas corretas com menor latência) seguiram a sequência que formava a palavra-solução. Após a passagem da Fase 1 para a Fase 2, esses participantes passaram a olhar os anagramas de modo a formar sequências diversas, mas rapidamente passavam a emitir a nova sequência de movimentos requerida para a formação da palavra-solução. O mesmo ocorreu após a mudança para a Fase 3. Kaplan e Schoenfeld interpretaram esses resultados como um condicionamento operante dos movimentos dos olhos. Este condicionamento pode ser descrito como: diante do anagrama, a resposta de mover os olhos na sequência que formava a palavra-solução produzia a palavra-solução e, diante da palavra-solução, a resposta de vocalizá-la produzia um reforçador, como talvez um elogio ou o próprio sucesso em resolver o anagrama.

$\mathrm{Na}$ literatura, os estudos sobre os movimentos dos olhos têm auxiliado a investigação de controle de estímulo (por exemplo, Dube et al., 1999; Dube et al., 2003; Endemann, 2008; Magnusson, 2002; Perez, 2008; Pessôa, Huziwara, Perez, Endemann, \& Tomanari, 2009; Schroeder, 1969a, 1969b, 1970, 1997). Nesse sentido, o rastreamento do olhar pode ser útil como fonte indicativa de controles de estímulos potencialmente envolvidos no comportamento textual, intraverbal etc. Considerando que estes comportamentos são classificados pelo controle da resposta por seus antecedentes, investigar experimentalmente os movimentos dos olhos na produção de estímulos antecedentes verbais pode auxiliar na compreensão das variáveis controladoras do tais comportamentos. Em termos de procedimento, o uso de anagramas, tal como utilizado pelos estudos descritos anteriormente (Rees \& Israel, 1963, citado por Kaplan \& Schoenfeld, 1966; Kaplan \& Schoenfeld, 1966) é estratégico, pois permite analisar correspondências discretas, ponto-a-ponto, entre estímulos visuais produzidos pelos movimentos dos olhos e as respostas orais que os seguem.

Revisões de literatura têm sugerido a necessidade de se ampliar não só a quantidade (Catania \& Shimoff, 1998; Norman, Fossa, \& Poling, 2000), mas também o escopo (Marcon-Dawson, Vicars, \& Miguel, 2009) da produção empírica baseada na proposta skinneriana (Skinner, 1957). Nesse sentido, a presente pesquisa replicou as fases primeira e segunda do grupo experimental de Rees e Israel (conforme citado por Kaplan \& Schoenfeld, 1966) com o objetivo de identificar os operantes verbais envolvidos na leitura dos anagramas tendo por base os movimentos dos olhos.

\section{Método}

\section{Participantes}

Dois adultos, um do sexo masculino e um do sexo feminino ( $\mathrm{P} 1$ e $\mathrm{P} 2$, respectivamente), ambos recém graduados, um em psicologia e um em biologia, participaram deste experimento. Os participantes leram e assinaram um "Termo de Consentimento Livre e Esclarecido".

\section{Ambiente Experimental e Equipamento}

A pesquisa foi realizada em uma sala 2,0 $\mathrm{m}$ x 3,5 m. Esta sala foi dividida por um biombo. De um lado do biombo havia uma cadeira e uma mesa com uma tela de computador que foram utilizadas pelos participantes. Do outro lado, os equipamentos utilizados pelos experimentadores, descritos abaixo.

Para registro das vocalizações e duração das apresentações, foram utilizados uma folha de registro e cronômetro. Para a elaboração e apresentação dos estímulos, foi utilizado o software PowerPoint ${ }^{\circledR}$. Como estímulos foram utilizados 30 slides de PowerPoint ${ }^{\mathbb{}}$ contendo um anagrama em cada um. Durante o experimento, os slides eram apresentados em um monitor Samsung modelo SyncMaster 950b de 17 polegadas, utilizando o mesmo software em modo Slide Show. Como mostra a Figura 1, cada anagrama era formado por cinco letras pretas maiúsculas, na fonte Arial, tamanho 60 , apresentadas sobre um fundo branco correspondente ao tamanho da tela. Como em Kaplan e Schoenfeld (1966) e Rees e Israel (conforme citado por Kaplan \& Schoenfeld, 1966), as cinco letras do anagrama foram apresentadas uma no centro (posição 3) e as demais nos quatro cantos do slide - canto superior esquerdo (posição 1), superior direito (posição 2), inferior esquerdo (posição 4) e inferior direito (posição 5). Nos 20 primeiros anagramas, as letras formavam apenas uma palavra, quando fixadas na sequência 3-4-5-2-1, como mostram as setas da Figura 1. Exemplos das palavras foram, vidro, ácido, mundo, horta, carne etc. Nos 10 últimos anagramas, as letras formavam mais de uma palavra, além daquela com a solução 3-4-5-2-1, por exemplo, porta (parto, prato). Estes 10 anagramas com mais de uma palavra-solução foram chamados de anagramas ambíguos. Para a participante P2, foi acrescentado um $31^{\circ}$ anagrama que, quando lido na sequência 1-2-3-4-5, formava a palavra FINAL. A distância entre as letras era, no mínimo, de $16 \mathrm{~cm}$ e a distância entre o olho do participante e a tela era de aproximadamente 60 $\mathrm{cm}$. Com isso, a distância angular ${ }^{2}$ entre as letras era de aproximadamente $11,4^{\circ}$.

Para o registro dos movimentos dos olhos, foi utilizado o equipamento de rastreamento do olhar ISCAN ${ }^{\circledR}$. Este equipamento é composto por uma micro-câmera de vídeo padrão, uma micro-câmera de vídeo sensível a luz infravermelha e por uma fonte de luz infravermelha. Estes três componentes são fixados em um suporte que se assemelha a uma viseira e estão conectados a duas placas de hardware, a RK-826PCI Pupil/Corneal Refletion Tracking System e a RK-630 Autocalibration System, instaladas em um computador PC com processador Pentium ${ }^{\circledR} 2 \mathrm{GHz}$ e $256 \mathrm{MB}$ de memória. O sistema também é composto pelo software ISCAN Raw Movement Data Acquisition. O ISCAN ${ }^{\circledR}$ contém uma saída de vídeo analógica, que é conectada a uma placa de captura de vídeo, instalada em outro computador PC, com processador Pentium $^{\circledR} 2$ GHz e 1 GB de memória RAM. Neste segundo computador foram instalados dois programas, o Pinnacle

2 Na situação experimental, porque o tamanho do estímulo exibido na tela do computador e projetado na retina do participante varia em função do tamanho absoluto e de sua distância ao olho, estudos envolvendo os movimentos oculares tradicionalmente convertem, por meio de cálculos trigonométricos, tais medidas absolutas (em centímetros, por exemplo) em medidas angulares. 
Studio Plus $9^{\circledR}$ e o Video Frame Coder, este segundo desenvolvido pela equipe da Universidade de Massachusetts Medical School - Shriver Center.

Assim que a viseira foi colocada na cabeça do participante, seu olho passou a ser iluminado pela fonte infravermelha. As áreas ao redor da pupila refletiram a luz infravermelha de volta para a câmera de vídeo sensível a esta luz, gerando imagens do olho com a "pupila escura". Por meio das imagens geradas pela câmera de vídeo sensível à luz infravermelha, a placa de hardware RK-826PCI monitora a posição da pupila e a marca da reflexão corneal em uma imagem de olho em tempo real.

A placa de hardware RK-630 integra as imagens geradas pela RK-826PCI às da segunda câmera de vídeo, que capta imagens do campo de visão do participante. Tal integração permite o cálculo do ponto central (fóvea) de fixação do olho do participante em relação à cena vista. Uma nova imagem é, então, gerada com o campo de visão do participante e com um cursor que indica o ponto central da fixação do olho em relação a este campo que, por sua vez, corresponde à imagem formada no centro da retina (fóvea), ou visão foveal.

O ISCAN Raw Movement Data Acquisition foi utilizado para controlar e calibrar o sistema e também para extrair, gravar e analisar os dados em relação ao diâmetro da pupila, às coordenadas horizontal e vertical do ponto de fixação do olhar e ao tempo decorrido desde o início da geração das imagens, em intervalos de aproximadamente 20 milissegundos.

O sinal de vídeo com o campo de visão do participante e com o cursor indicando o ponto central da fixação do olho (fóvea) em relação a este campo foi capturado no segundo computador através da placa de captura de vídeo e do software Pinnacle Studio Plus $9^{\circledR}$ e gravado em arquivos com formato AVI. A análise dos arquivos gravados foi realizada com o software Video Frame Coder. Por meio deste software, é possível avançar o vídeo quadro-a-quadro e codificar, por meio de letras ou números, cada um desses quadros. Isso possibilita que seja registrada a posição do cursor gerado pelo ISCAN $®$ em relação aos estímulos apresentados pelo experimentador ou a qualquer outro evento filmado pela micro-câmara padrão. A partir desses códigos, o programa gera automaticamente uma planilha eletrônica com o número do quadro, o tempo de sua ocorrência e o código correspondente. A partir dessa planilha, são calculadas, por exemplo, a frequência e a duração do olhar para os estímulos.

Antes do início das sessões, foi realizada uma rotina de calibragem do equipamento. $\mathrm{O}$ experimentador instruía o participante a olhar para (fixar) $)^{3}$ diferentes pontos na tela do computador, enquanto o equipamento registrava as coordenadas do cursor (indicativo de onde o participante olha) dentro do campo visual. A partir dessas coordenadas, tem-se a correspondência exata entre o cursor e os movimentos e fixação dos olhos do participante. De modo a conferir a estabilidade da calibragem, antes do início e ao final de cada sessão (as sessões experimentais serão descritas adiante), o participante era instruído a fixar novamente os diferentes pontos na tela do computador. Ao final da sessão, caso a calibragem fosse

3 O termo "fixação" corresponde à formação da imagem na retina (Becker, 1991; Schwarts, 2004). "Fixar" uma imagem é sinônimo de "olhar para", "observar" e "focalizar". considerada inadequada (o que não foi o caso), o vídeo teria sido descartado.

\section{Procedimento}

Uma vez calibrado o equipamento, a sessão experimental era iniciada pela leitura da seguinte instrução para o participante: "Por favor, ache as palavras formadas pelas letras apresentadas nos slides. Cada slide ficará exposto por 20 segundos ou até você falar uma palavra".

Cada participante foi exposto à sessão experimental individualmente na presença de dois experimentadores. A sessão experimental foi composta de duas fases consecutivas e ininterruptas. A Fase 1 foi composta por 20 apresentações. Cada apresentação durava no máximo 20 s ou até a vocalização de uma palavra. Em cada anagrama, havia apenas uma palavra-solução, aquela formada pelas letras ordenadas na sequência 3-4-5-2-1 (Figura 1). As vocalizações corretas dos participantes foram consequenciadas intermitentemente em VR 2 por um dos experimentadores, que dizia "certo". $\mathrm{O}$ uso de um esquema intermitente durante a Fase 1 foi utilizado tanto para fortalecer as vocalizações das palavras na seqüência ensinada, como também, para minimizar possíveis efeitos da não apresentação daquela consequência na fase seguinte. Imediatamente após a vocalização do participante, um slide em branco era apresentado. Durante a apresentação desse slide em branco, um dos experimentadores registrava a palavra vocalizada e, por meio de um cronômetro, o intervalo de tempo transcorrido entre apresentação do anagrama e a vocalização da palavra-solução (latência da resposta). Após a realização dos registros, o próximo anagrama era apresentado.

Após a apresentação dos 20 anagramas com apenas uma palavra-solução (Fase 1), era iniciada a Fase 2, na qual 10 anagramas ambíguos eram apresentados sucessivamente. $\mathrm{Na}$ Fase 2, o procedimento de apresentação dos anagramas e o registro das respostas foram idênticos aos da Fase 1. No entanto, nessa fase, as vocalizações dos participantes não eram consequenciadas pelo experimentador.

Em ambas as fases, foram registradas as palavras vocalizadas, a latência da resposta de vocalização e os movimentos dos olhos dos participantes em relação às letras dos anagramas.

$\mathrm{O}$ acordo entre os dois observadores que registraram as letras fixadas pelos participantes, utilizando-se o método de sessão inteira (Repp, Deitz, Boles, Deitz, \& Repp, 1976), foi de $92 \%$ de concordância entre os registros de P1 e de $94 \%$ de concordância entre os registros de P2.

\section{Resultados}

\section{Respostas de Vocalização da Palavra-Solução}

O participante P1 vocalizou a palavra-solução diante de todos os anagramas, tanto da Fase 1 quanto da Fase 2, exceto diante do quinto anagrama apresentado, que continha a palavra ÁCIDO. Diante deste anagrama, P1 não vocalizou 
nenhuma palavra. A participante P2 vocalizou a palavra-solução diante de todos os anagramas.

O intervalo de tempo entre a apresentação do anagrama e a vocalização das palavras-solução, ou seja, a latência da resposta dos participantes, pode ser observada na parte inferior da Figura 2. Nesta figura, pode ser observada, a partir do sexto anagrama para P1 e do quarto para P2, uma queda na latência das respostas. Para P1, até o sexto anagrama, a latência média das respostas foi de $11,7 \mathrm{~s}$, variando entre 3,9 s e 20,3 s. A partir do sexto anagrama até o fim da sessão, a latência média foi de 3,1 s, variando entre $1,72 \mathrm{~s}$ e 3,99 s. Para P2, até o quarto anagrama, a latência média foi de 4,44 s, variando entre 2,72 s e 6,49 s. A partir do quarto anagrama até o penúltimo (trigésimo), a latência média foi de 2,5 s, variando entre 1,57 s e 3,48 s. No último anagrama, em que foi apresentada a palavra FINAL, formada por uma sequência distinta dos demais anagramas, a latência da resposta foi de 4,39s.

\section{Fixações e Movimentos dos Olhos sobre as Letras do Anagrama}

A queda nas latências da vocalização da palavra-solução acompanhou a queda do número de letras fixadas (ver Nota de Rodapé 2) ao longo da apresentação dos anagramas. A parte superior da Figura 2 apresenta, para ambos os participantes, o número de letras fixadas ao longo das apresentações, levando em consideração as repetições, inclusive repetições consecutivas, das letras.

Para o participante $\mathrm{P} 1$, até o sexto anagrama, o número de letras fixadas variou entre 9 e 44. A partir do sexto anagrama, até o fim da sessão, o número de letras fixadas variou entre 6

P1
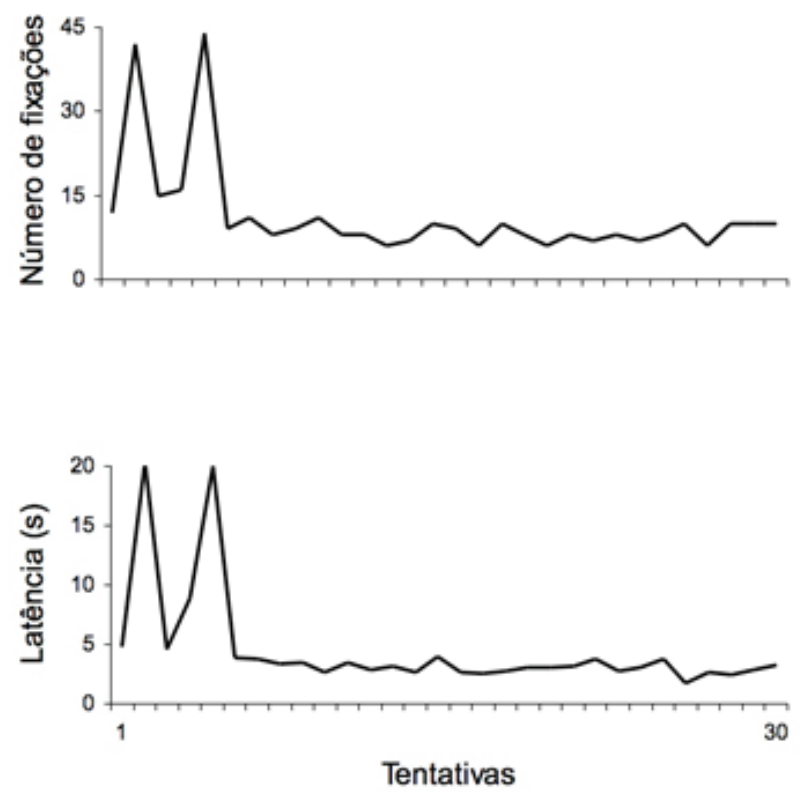

e 11. Para o participante $\mathrm{P} 2$, até o quarto anagrama, o número de letras fixadas variou entre 6 e 11. O dado do desempenho do participante na primeira apresentação foi perdido. A partir do quarto anagrama, até o penúltimo (trigésimo), o número de letras fixadas variou entre 6 e 7 . No último anagrama, cuja palavra era formada por uma sequência distinta, 18 letras foram fixadas.

\section{Sequência dos Movimentos dos Olhos sobre as Letras do Anagrama}

A Figura 3 apresenta a frequência acumulada da ocorrência da sequência de fixação 3-4-5-2-1. Por exemplo, diante do anagrama que continha a palavras MUNDO, caso a sequência de letras fixadas fosse M-D-M-U-N-D-O-N ou M-U-N-D-O, em ambos os casos, registrou-se a ocorrência da sequência 3-4-5-2-1, que corresponde à fixação das letras na sequência que forma a palavra-solução. Por outro lado, diante do mesmo anagrama, caso a sequência de letras fixadas fosse M-U-M-N-D-O, por exemplo, embora as letras da sequência que forma a palavra-solução pudessem ser identificadas nesse conjunto, não ocorria o registro, posto que era considerada apenas a fixação das letras na sequência 3-4-5-2-1 sem interrupção.

Ao longo dos anagramas, ambos os participantes passaram a fixar as letras na sequência que formava a palavra-solução (3-4-5-2-1). Já no primeiro e, mais frequentemente, do sétimo anagrama em diante, $\mathrm{P} 1$ fixou as letras na sequência que formava a palavra-solução. Para esse participante, no $15^{\circ}, 18^{\circ}$ e $28^{\circ}$ anagrama, a sequência 3-4-5-2-1 foi emitida duas vezes. Para P2, a fixação das letras na sequência que formava a palavra-solução ocorreu mais frequentemente do quarto anagrama em diante.

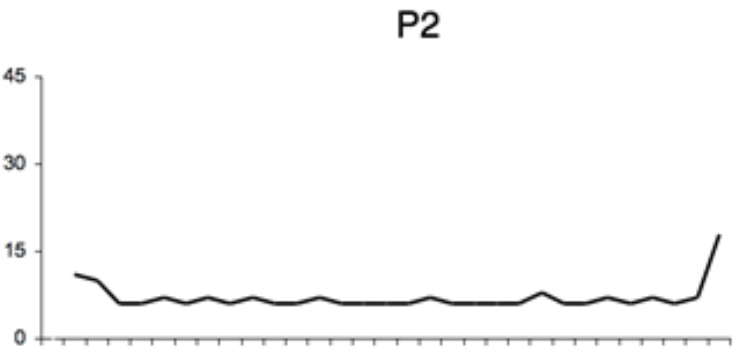

Figura 2. Número de fixações (gráficos acima) e latência (em segundos) das respostas de vocalização da palavra-solução (gráficos abaixo) ao longo das tentativas, para os participantes P1 (gráficos à esquerda) e P2 (gráficos à direita). 


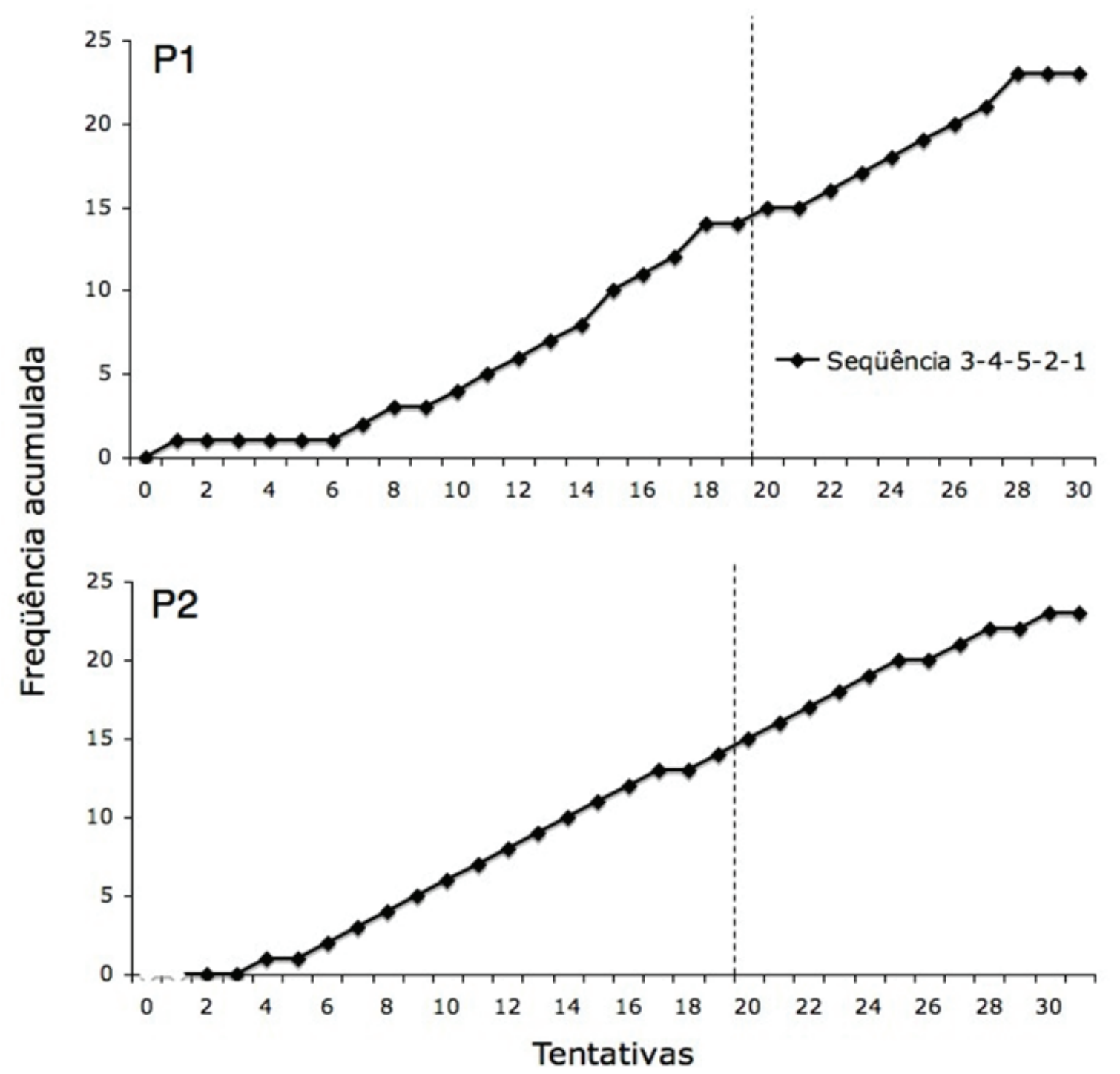

Figura 3. Freqüência acumulada da seqüência de fixação 3-4-5-2-1 em cada uma das apresentações dos anagramas (tentativas).

\section{Relação entre Vocalizações e Movimentos dos Olhos}

A análise dos dados mostrada na Figura 3 diz respeito às situações em que os olhos fixaram-se sobre as letras que, na sequência, formavam a palavra corretamente vocalizada. Entretanto, houve casos em que a palavra-solução foi vocalizada sem que a sequência que a formava ocorresse de forma ininterrupta (por exemplo, M-N-U-D-N-D-M-O) ou não ocorresse no conjunto total das fixações (por exemplo, N-D-O-D-U-M). Esses casos são apresentados na Tabela 1.
A primeira coluna da Tabela 1 apresenta a frequência absoluta de ocorrência de fixação das letras na sequência 3-4-5-2-1 de modo ininterrupto. A segunda, terceira e quarta colunas apresentam a ocorrência de outras sequências em que as letras das posições 3, 4, 5, 2 e 1 são fixadas nessa ordem, porém, interrompidas por outras fixações. Como no exemplo acima, $M-\mathrm{N}-U-\mathrm{D}-\mathrm{N}-\mathrm{D}-\mathrm{M}-O$ (3-5-4-2-5-2-3-1), em que ocorrem três interrupções (N, D e M). Na segunda, terceira e quarta colunas, é apresentada a ocorrência de duas, três e quatro interrupções, respectivamente. Na quinta coluna, é apresentada a ocorrência de sequências de fixação das letras

Tabela 1. Tipos diferentes de produção de estímulo antecedente à vocalização, por participante. Os números da tabela fazem referência ao número de vezes que a palavra-solução foi vocalizada corretamente com diferentes sequências de fixação das letras.

\begin{tabular}{ccccccc}
\hline \multirow{2}{*}{ Participantes } & $\begin{array}{c}\text { Sequência } \\
3-4-5-2-1\end{array}$ & interrupção & interrupções & interrupções & $\begin{array}{c}\text { Outras } \\
\text { sequências }\end{array}$ & $\begin{array}{c}\text { Total } \\
\text { P1 }\end{array}$ \\
\hline P2 & 21 & 3 & 1 & 1 & 3 & 29 \\
\hline
\end{tabular}


em que a sequência 3-4-5-2-1 não ocorreu (como no exemplo acima, N-D-O-D-U-M).

O total de apresentações de anagramas para P1 na Tabela 1 é 29 porque o participante não vocalizou a palavra-solução no anagrama 5 (Fase 1). O total de apresentações para P2 é 30 porque o registro dos movimentos dos olhos durante a primeira apresentação (Fase 1) foi perdido.

Finalmente, para P2, no último anagrama, a sequência da palavra-solução (FINAL) apareceu com seis interrupções e com quatro fixações anteriores à primeira letra $(F)$ e mais três fixações após a última letra (L). É preciso lembrar que esta última palavra-solução foi apresentada em sequência diferente das apresentadas até então.

\section{Resposta de Vocalização e Sequências de Fixação na Fase 2}

Com a introdução dos anagramas ambíguos na Fase 2, ambos os participantes mantiveram o padrão de movimento dos olhos apresentado na Fase 1 (Figura 3). Na Fase 2, ambos os participantes vocalizaram a palavra-solução da sequência 3-4-5-2-1, a despeito da possibilidade de que outras palavras pudessem ser formadas por outras sequências.

\section{Discussão}

O presente estudo replicou os resultados de Rees e Israel (conforme citado por Kaplan \& Schoenfeld, 1966) e de Kaplan e Schoenfeld (1966) na medida em que, comparando-se a Fase 1 com a Fase 2, foi possível observar o condicionamento operante dos movimentos dos olhos e da vocalização da palavra-solução na sequência 3-4-5-2-1. Além disso, foram produzidos dados que possibilitaram identificar operantes verbais envolvidos na leitura dos anagramas tendo por base os movimentos dos olhos na produção de estímulos antecedentes para o comportamento verbal.

\section{Movimentos dos Olhos e Vocalização da Palavra- Solução}

A partir de uma disposição espacial das letras diferente daquela comumente apresentada em textos como livros, revistas etc., e com a ajuda do equipamento de rastreamento dos movimentos dos olhos, foi observado que a resposta de movimentar os olhos na sequência 3-4-5-2-1 foi selecionada na Fase 1. Essa resposta se manteve na Fase 2 (Figura 3 ), apesar da possibilidade de que outras palavras fossem formadas com o mesmo conjunto de letras. Esse resultado replica os resultados de Rees e Israel relatados por Kaplan e Schoenfeld (1966).

Na Figura 2, foi observado que as maiores latências das respostas de vocalização ocorreram nos anagramas em que houve um maior número de fixações. A sequência 3-4-5-21 foi a que mais rapidamente produziu a palavra-solução em comparação com as demais sequências. Deste modo, a sequência 3-4-5-2-1 possibilitou um menor tempo entre a apresentação do anagrama e a apresentação do elogio. Este fato pode ter contribuído para o condicionamento operante dos movimentos dos olhos, pois mesmo que a emissão de outros movimentos também produzisse a ocasião para a vocalização da palavra-solução, os participantes passaram a emitir com alta frequência os movimentos que compunham a sequência 3-4-5-2-1.

\section{Movimentos dos Olhos na Produção de Estímulos Antecedentes para o Comportamento Verbal}

Como dito anteriormente, Skinner (1957) descreve o comportamento textual como aquele em que respostas vocais têm como estímulo antecedente um conjunto de letras, palavras ou um texto e são mantidas pela apresentação de reforçador generalizado. Ainda, por definição, no comportamento textual, deve ocorrer uma correspondência ponto-a-ponto entre os estímulos discriminativos verbais e a resposta de vocalização que é ocasionada por aqueles estímulos. Nos casos em que as respostas de vocalização da palavra-solução foram antecedidas pelo estímulo "sequência 3-4-5-2-1", foi possível identificar a correspondência ponto-a-ponto entre os estímulos antecedentes e a resposta de vocalização, cumprindo-se claramente os critérios que definem o comportamento textual.

Por outro lado, houve casos em que a palavra-solução foi vocalizada sem a produção do estímulo "sequência 3-45-2-1" (Tabela 1). No caso do presente experimento, apenas este estímulo permitiria a correspondência ponto-a-ponto entre um antecedente impresso e a resposta de vocalização, caracterizando um comportamento textual.

Ao recuperar a discussão de Skinner (1957) acerca das unidades de controle do comportamento verbal, de Rose (2005) explicita dinâmicas do comportamento operante. Segundo de Rose, "unidades moleculares [de comportamento] podem encadear-se, integrando-se em unidades molares (globais); unidades molares podem, por sua vez, ser fracionadas em unidades mais moleculares." (p. 37). No caso do presente experimento, a unidade molar referida por de Rose seria uma unidade composta por "anagrama, vocalização da palavra-solução e elogio". Essa unidade levaria a considerar-se todos os casos do presente experimento como casos de comportamento textual. Porém, a disposição das letras em anagramas e o registro do movimento dos olhos possibilitaram a análise de unidades moleculares de comportamento que formaram a unidade global. Essas unidades moleculares encadeiam-se na formação da unidade maior. Em um primeiro momento, o anagrama poderia não ter funcionado como estímulo discriminativo para a emissão da palavra-solução. Porém, pode ter havido o controle de unidades menores do que a palavra, por exemplo, algumas letras ou sílabas e, a partir de respostas emitidas sob controle dessas unidades, foi possível finalmente vocalizar a palavra-solução. Ao tratar do comportamento textual, Skinner (1957) afirma que:

\footnotetext{
"Alguma autocorreção é possível em amostras mais amplas de comportamento textual. Pode-se responder, primeiramente, com uma sílaba, uma palavra ou uma frase deturpada, então, mudar para uma forma correta que "soe adequada" ou que "faça sentido". Isso depende de um condicionamento anterior da resposta
} 
do ouvinte, e a resposta usualmente "soa adequada" ou "faz sentido" somente se ela é de tamanho substancial" (p. 69)4.

Na citação acima, Skinner se refere a "amostras mais amplas do comportamento textual". Como verificado na Tabela 1, aproximadamente um quarto das 59 vocalizações das palavras-solução não foi antecedida por movimentos dos olhos que produziriam um estímulo formado pela ordem exata das letras. Nesse um quarto dos anagramas, talvez possa ter havido autocorreção. Como Skinner diz na citação acima, na autocorreção pode haver mais de uma resposta envolvida. Uma resposta produz um estímulo verbal "com uma sílaba, uma palavra ou uma frase deturpada" e a outra, posterior, produz o estímulo verbal que "soe adequado" ou que "faça sentido". No presente experimento, a descrição do comportamento a partir dos movimentos dos olhos talvez permita descrever a autocorreção. Por exemplo, movimentos dos olhos de P2 produziram o estímulo POATEAP. Esse estímulo pode ter controlado a resposta vocal (aberta ou encoberta) "poateap". Por sua vez, o estímulo "poateap" controlou a verbalização da palavra que "fazia sentido" ("poeta"). Como acrescenta Skinner, a palavra que faz sentido "depende do condicionamento prévio da resposta do ouvinte". No presente experimento, participaram leitores proficientes da língua portuguesa, que foram apresentados à instrução "ache uma palavra" e expostos a anagramas com cinco letras formando uma única palavra da língua portuguesa (Fase 1). Todas essas condições possibilitaram a autocorreção.

Uma segunda questão que pode ser levantada é se o comportamento de vocalizar a palavra-solução após a produção de uma sequência distinta de 3-4-5-2-1 era, de fato, um comportamento textual. No caso do presente experimento, diante do anagrama, os movimentos dos olhos podem ter produzido estímulos verbais que ocasionaram outro comportamento verbal, o intraverbal. O comportamento intraverbal é aquele em que a resposta verbal é controlada por um estímulo verbal antecedente sem haver correspondência ponto-a-ponto entre eles. E ainda, segundo de Rose (2005), intraverbais podem possibilitar a antecipação da resposta verbal. Por exemplo, diante da sequência V-N-M-U-E-M-N-U, o encadeamento das duas últimas letras $(\mathrm{N}-\mathrm{U})$ pode ter controlado a vocalização da palavra "nuvem".

As condições produzidas pelos movimentos dos olhos e discutidas a partir de relações intraverbais continham maior número de letras fixadas por um tempo maior do que aquelas comprometidas na identificação do comportamento textual. No caso desse experimento, a antecipação da resposta verbal não coincidiu com as respostas com menores latências.

Levando em consideração que os participantes estavam diante de palavras em um novo arranjo espacial das unidades, a palavra-solução deveria ser vocalizada sob controle também das outras letras do anagrama. Dessa forma, é possível que a vocalização "nuvem" diante da sequência V-N-M-U-E-M-N-U tenha sido ocasionada pelas duas últimas

4 Some self-correction is possible in larger samples of textual behavior. One may respond first with a garbled syllable, word, or phrase and then change to a correct form which "sounds right" or "makes sense." This depends upon the prior conditioning of the response of the listener, and a response usually "sounds right" or "makes sense" only if it is of substantial size (Skinner, 1957, p. 69). letras produzidas em interação com a instrução fornecida ("ache uma palavra"), o contato com as demais letras e com a história de reforçamento com outros anagramas (todos com cinco letras, formando uma palavra). Deste modo, os movimentos dos olhos que produziram estímulos (sequências diversas) para a emissão do comportamento intraverbal foram de maior duração e com maior número de fixações às letras do que aquelas que produziam estímulos para a emissão do comportamento textual (sequência 3-4-5-2-1).

Até o presente momento da discussão, as vocalizações foram analisadas à luz de dois operantes verbais - textual e intraverbal. Para cada sequência de letras produzidas pelos movimentos dos olhos, um operante verbal distinto foi atribuído à vocalização. Outra questão é se, para uma única vocalização, podem ser identificadas características da ocorrência de mais de um operante verbal. Segundo de Rose (2005),

\begin{abstract}
"é mais conveniente analisar a leitura e escrita não como comportamentos unitários, mas como repertórios envolvendo um conjunto de comportamentos distintos. O objetivo de uma análise comportamental é a identificação destes componentes dos repertórios de leitura e escrita, e uma descrição de suas relações características” (p. 30).
\end{abstract}

Segundo Skinner (1957), "o comportamento verbal é usualmente o efeito de múltiplas causas. Variáveis separadas são combinadas para estender seu controle funcional e novas formas de comportamento emergem da recombinação de antigos fragmentos.” (p. 10). Deste modo, é possível analisar as vocalizações, que foram antecedidas pelos estímulos diferentes da "sequência 3-4-5-2-1", como instâncias multicausadas. Como afirma de Rose (2005),

\begin{abstract}
"à medida que a pessoa aprende a ser ouvinte de seu próprio comportamento textual, a leitura passa também a ficar parcialmente sob controle das respostas textuais antecedentes. Essas relações intraverbais (cf. Skinner, 1957) permitem que o leitor 'antecipe' a sequência de um texto utilizando apenas parte das pistas textuais" (p. 10).
\end{abstract}

Quando os movimentos dos olhos formaram, por exemplo, o estímulo MUN, esse estímulo foi condição antecedente para a emissão da resposta vocal (aberta ou encoberta) "mun", sendo possível identificar, nesta relação, correspondência ponto-a-ponto. Por sua vez, o estímulo "mun" ocasionou a resposta intraverbal "do", o que, em uma unidade molar, formou a palavra-solução "mundo". Parte da vocalização ficou sob controle textual, ou seja, sob controle de um estímulo discriminativo verbal escrito, mantendo correspondência ponto-a-ponto e parte, sob controle dos estímulos verbais antecedentes, o que permitiu a antecipação e por isso uma relação intraverbal. Deste modo, vocalizar a palavra diante de sequências diversas, produzidas pelos movimentos dos olhos, pôde ser decomposto por apresentar determinantes definidores de mais de um operante verbal.

Estas análises foram possíveis a partir do rastreamento dos movimentos dos olhos e dos estímulos por eles produzidos. Segundo Skinner (1957), "para conhecer o comportamento de modo eficaz, devemos conhecer as circunstâncias 
nas quais ele é emitido" (p. 186). Assim, o equipamento de rastreamento dos olhos permitiu a identificação e discussão de possíveis variáveis que atuaram para a formação de fragmentos do comportamento verbal a partir das unidades de controle exercido pelos estímulos antecedentes. Futuras pesquisas poderiam programar a apresentação de anagramas com sílabas ou fonemas. Isso talvez permita uma maior aproximação entre os estímulos produzidos pelo olhar e as unidades de controle da vocalização da palavra. Além disso, o uso de sílabas aproximaria a unidade de análise dos movimentos dos olhos à unidade de análise que tem sido utilizada em outros estudos (por exemplo, de Rose et al., 1996; de Souza et al., 2009; Hübner et al., 2009)

Em resumo, neste experimento, a análise do comportamento verbal a partir dos movimentos dos olhos permitiu verificar a ocorrência de dois casos de controle de estímulo para a resposta de vocalização. No primeiro caso, houve a produção de estímulos antecedentes que permitiram a análise das vocalizações por meio da correspondência ponto-a-ponto. Foi caracterizado, deste modo, o comportamento textual. Ainda, nesses casos, de emissão de comportamento textual, houve um número relativamente menor de fixações e uma latência menor da vocalização quando comparado ao segundo caso. No segundo caso, em que não houve apenas correspondência ponto-a-ponto, algumas discussões foram possíveis, envolvendo autocorreção, intraverbais ou relações intraverbais/textuais na composição da vocalização.

\section{Referências}

Becker, W. (1991). Saccads. In J. R. Cronly-Dillon (Ed. da série) \& R. H. S. Carpenter (Ed. do volume), Vision and visual dysfunction: Vol. 8. Eye movements (1 ${ }^{\mathrm{a}}$ ed., pp. 1-339). New York: The McMillan Press Ltd.

Catania, C., \& Shimoff, E. (1998). The experimental analysis of verbal behavior. The Analysis of Verbal Behavior, 15, 97-100.

de Rose, J. C. C. (2005). Análise comportamental da aprendizagem de leitura e escrita. Revista Brasileira de Análise do Comportamento, 1, 1-32.

de Rose, J. C., de Souza, D. G., \& Hanna, E. S. (1996). Teaching reading and spelling: Exclusion and stimulus equivalence. Journal of Applied Behavior Analysis, 29, 451-469.

de Souza, D. G., de Rose, J. C., Faleiros, T. C., Bortoloti, R., Hanna, E. S., \& McIlvane, W. J. (2009). Teaching generative reading via recombination of minimal textual units: A legacy of verbal behavior to children in Brazil. Revista Internacional de Psicología y Terapia Psicológica, 9, 19-44.

Dube, W. V., Lombard, K. M., Farren, K. M., Flusser, D. S., Balsamo, L. M., \& Fowler, T. R. (1999). Eye tracking assessment of stimulus overselectivity in individuals with mental retardation. Experimental Analysis of Human Behavior bulletin, 17, 8-14.

Dube, W. V., Lombard, K. M., Farren, K. M., Flusser, D. S., Balsamo, L. M., Fowler, T. R., \& Tomanari, G. Y. (2003). Stimulus overselectivity and observing behavior in individuals with mental retardation. Em S. Soraci Jr. \& K. Murata-Soraci (Eds.), Visual information processing (pp. 109-123). London: Proeger.
Endemann, P. (2008). Resposta de observação e movimento dos olhos em uma situação de discriminação simples simultânea. Dissertação de Mestrado, Instituto de Psicologia, Universidade de São Paulo, São Paulo, Brasil. Disponível em: http://www. teses.usp.br/teses/disponiveis/47/47132/tde-01122009103022/en.php

Gompel, R. P. G. V., Fischer, M. H., Murray, W. S., \& Hill, R. L. (2007). Eye movements: A window on mind and brain. Amsterdam: Elsevier

Hübner, M. C., Gomes, R. C., \& McIlvane, W. J. (2009). Recombinative generalization in minimal verbal unit-based reading instruction for pre-reading children. Experimental Analysis of Human Behavior Bulletin, 27, 11-17.

Kaplan, I., \& Schoelfeld, W. N. (1966). Oculomotor paterns during the solution of visually displayed anagrams. Journal of Experimental Psychology, 72, 447-451.

Magnusson, A. (2002). Topography of eye movements under select and reject control. Dissertação de mestrado não publicada. Boston: Northeastern University.

Marcon-Dawson, A., Vicars, S. N., \& Miguel, C. F. (2009). Publication trends in The Analysis of Verbal Behavior: 19992008. The Analysis of Verbal Behavior, 25, 123-132.

Matos, M. A. (1991). As categorias formais de comportamento verbal em Skinner. Anais da XXI Reunião Anual da Sociedade de Psicologia de Ribeirão Preto, Brasil, 333-341.

Norman, M. P., Fossa, J. F., \& Poling, A. (2000). Publication trends in The Analysis of Verbal Behavior: 1982-1998. The Analysis of Verbal Behavior, 17, 167-173.

Passos, M. L. R. F. (2003). A análise funcional do comportamento verbal em Verbal Behavior (1957) de B. F. Skinner. Revista Brasileira de Terapia Comportamental e Cognitiva, 5, 195-213.

Passos, M. L. R. F (2007). Skinner's definition of verbal behavior and the arbitrariness of the linguistic signal. Temas em Psicologia, 15, 161-172.

Pessôa, C., Huziwara, E., Perez, W. F., Endemann, P., \& Tomanari, G. Y. (2009). Eye fixations to figures in a four-choice situation with luminance balanced areas: Evaluating practice effects. Journal of Eye Movements Research, 2, 1-6. Acessado em 08 de julho de 2010, em http://www.jemr.org/online/2/5/3

Perez, W. F. (2008). Movimentos dos olhos e topografias de controle de estímulo em treinos de discriminação condicional e testes de equivalência. Dissertação de Mestrado, Instituto de Psicologia, Universidade de São Paulo, São Paulo, Brasil.

Repp, A. C., Deitz, D. E. D., Boles, S. M., Deitz, S. M., \& Repp, C. F. (1976). Differences among common methods for calculating interobserver agreement. Journal of Applied Behavior Analysis, 9, 109-113.

Schroeder, S. R. (1969a). Effects of cue factors on selective eye movements and choices during successive discriminations. Perceptual and Motor Skills, 29, 991-998.

Schroeder, S. R. (1969b). Fixation and choice selectivity during discrimination transfer. Psychonomic Science, 17, 324-325.

Schroeder, S. R. (1970). Selective eye movements to simultaneously presented stimuli during discrimination. Perception \& Psychophysics, 7, 121-124.

Schroeder, S. R. (1997). Selective eye fixations during transfer of discriminative stimulus control. Em D. M. Baer \& E. M. Pinkston (Eds.), Environment and Behavior (pp. 97-110). Boulder, CO: Westview. 
Schroeder, S. R., \& Holland, J, G. (1968a). Operant control of eye movements. Journal of Applied Behavior Analysis, 1, 161-166.

Schroeder, S. R., \& Holland, J, G. (1968b). Operant control of eye movements during human vigilance. Science, 161, 292-293.

Schwartz, S. H. (2004). Visual Perception - a clinical orientation. New York: McGraw Hill.

Skinner, B. F. (1957). Verbal Behavior. New Jersey: Prentice Hall.
Recebido em 06.11.2009

Primeira decisão editorial em 11.06.2010

Versão final em 12.07.2010

Aceito em 20.07.2010

\section{CONGRESSO IBEROAMERICANO DE AVALIAÇÃO PSICOLÓGICA}

Período: 14 de Abril - 16 Abril 2011

Local: Lisboa, PT

X INTERNATIONAL CONFERENCE THE ANCESTORS IN PERSONAL, PROFESSIONAL AND SOCIAL HISTORY

Período: 12 de Maio - 15 Maio 2011

Local: St Petersburg, SU 